\title{
Oxidative Stress Index (OSI) Condensed Questionnaire
}

\author{
Harold I. Zeliger
}

\begin{abstract}
The Oxidative Stress Index (OSI) has been shown to predict the onset of non-communicative disease. A condensed form of the OSI questionnaire has now been developed to enable meaningful OSI data to be obtained from answers to only eight questions, rather than from the $400+$ items contained in the original detailed questionnaire.
\end{abstract}

Index Terms-Disease onset mechanism; Environmental disease cause; Oxidative stress and disease; Predicting disease onset.

\section{INTRODUCTION}

Elevated oxidative stress (OS) is well known to be a direct cause of disease [Kelly, 2003; Zeliger 2016; Riggs, et al., 2020] and the oxidative stress index (OSI) has been shown to be an indicator of the likelihood of noncommunicable disease onset [Zeliger, 2017]. The OSI can also be used in multiple other applications, including predicting disease causing parameters for Alzheimer's disease and other diseases [Zeliger, 2019], as a public health survey instrument [Zeliger, 2019a], predicting adverse drug reactions [Zeliger, 2019a] and as a health prescreening tool and for health screening in areas with limited medical facilities and personnel [Zeliger, 2017].

The detailed OSI is based upon responses to a detailed questionnaire that address all contributory items to a person's OS level. In addition to being a valuable predictor of disease onset likelihood, it is also an indicator of lifestyle changes that can be made to lower OS and thereby help prevent disease. The OSI addresses genetics, age, weight, illnesses, conditions that are exacerbated by increased OS, medical symptoms, clinical laboratory results, prescription medications regularly taken, lifestyle and emotional stress.

The length of the detailed OSI questionnaire (containing more than 400 items), however, does not readily lend itself to regular use in clinical settings. Presented here and shown in table 1 is a condensed form of the OSI questionnaire, containing only eight parts, which can be readily used as a screening device to indicate the OSI level. Analysis has shown that OSI values obtained from the condensed OSI form produce scores that are nearly identical to those obtained from the original detailed OSI form. Accordingly, the condensed form can serve as a preliminary screening device to flag potential problems. Those with elevated OSIs in the condensed form can then use the detailed form to help identify specific causes of elevated OS. This can lead to clinically relevant follow up and preventive measures. The modified, currently used, detailed OSI form is shown in Table 2.

Published on February 24, 2020.

H. I. Zeliger is with Zeliger Research, LLC, 41 Wildwood Drive, Cape Elizabeth, Maine, USA. (email: hiz@zeliger.com)
The eight items in the condensed form are; age, personal information, prevalent diseases, medications taken, genetics, education level, place of residence and psychological stress. As discussed below, these include all major sources of OS elevation and produce OSI values that are within 90 percent of those obtained using the detailed OSI questionnaire.

\section{MEthodS}

The OSI condensed form is based upon health impacts of the various parameters as reported in the literature. The scoring assignments for the individual parameters in the condensed form are based upon experience with the detailed OSI form. The point values assigned to each parameter are based upon those obtained via the detailed OSI questionnaire.

\section{RESUlts AND DiscUSSION}

\section{A. Age}

Aging is accompanied by an increase in OS, generally starting around age 40 and continuing throughout life [Epel, et al., 2004; Hou, et al., 2015]. Accordingly, one OSI point is scored for each decade. For one aged 40-49, one OSI point is added to his or her total, 2 points are added for those aged 50-59, etc. [Zeliger, 2017].

\section{B. Personal}

Being overweight is associated with elevated OS and the onset of numerous diseases. Excessive weight is a worldwide health issue that continues to grow. In the United States, more than 70 percent of adults are overweight, more than half of whom are obese [CDC, 2018]. Diseases associated with excess weight include, but are not limited to; hypertension, dyslipidemia, type 2 diabetes, coronary heart disease, stroke, gallbladder disease, osteoarthritis, sleep apnea, breathing difficulties, anxiety, depression and several cancers (endometrial, breast, colon, kidney, liver and gallbladder [CDC, 2015]. Gender, height and weight information enables one to determine if the person is of normal weight, is heavy, or obese. The original detailed OSI addresses weight impact by number of pounds a person is overweight [Zeliger, 2017. The condenses OSI categorizes weight into three categories; normal, overweight and obese. Normal weight is assigned a value of zero. Heavy individuals are assigned a score of 1 and obese people are assigned a score of 2 .

\section{Chronic Prevalent Diseases}

The number of prevalent diseases at the time of OSI measurement is a critical indicator of OS. It is well established that disease increases total OS and hence the OSI [Zeliger, 2016; Zeliger 2017]. All diseases have 
symptoms associated with them. These are individually addressed in the detailed OSI. The OSI condensed form does not probe individual symptoms. Rather, it has been found that assigning five points for each prevalent disease adequately incorporates elevations in OS associated with the prevalent diseases. Respondents are asked to list all diseases they currently have been diagnosed with. Multiplying the number of prevalent diseases by five yields the disease number in the condensed OSI.

\section{Medications}

Essentially all pharmaceutical medications raise OS [Zeliger, 2017] and all have adverse drug reactions associated with them [Zeliger, 2019b]. The detailed OSI form addresses these individually. In the condensed form, one OSI point is assigned for each medication taken.

\section{E. Genetics}

Genetics is well known to be a factor in most noncommunicable diseases [Dato, et al., 2013; Jiang, et al., 2013; Guillaumet-Adkins, et al., 2017]. Indeed, many diseases such including Alzheimer's disease, Parkinson's disease and cancers, just to name and few, "run in families." Recently, epigenetics, as well, has been shown to lead to heritable diseases [Cencioni, et al., 2013; GuillaumetAdkins, et al., 2017]. Though all non-communicable diseases are more prevalent in those whose ancestors have suffered from those diseases, parental disease is most closely associated with the likelihood of disease onset in an individual [Awdeh, et al., 2006]. Both genetic traits and epigenetic effects raise OS [Cencioni, et al., 2013; Dato, et al., 2013; Jiang, et al., 2013; Guillaumet-Adkins, et al., 2017]. In the condensed OSI form, respondents are asked to check which of their prevalent diseases were also prevalent in their parents and each genetic limk is assigned a value of one OSI point.

\section{F. Education}

Socioeconomic status (SES) is well established as an indicator of detrimental lifestyle choices that raise OS [Mielck, et al., 2014]. These lifestyle choices include diets high in fats, sugar, salt or processed foods, tobacco, alcohol and recreational drug abuse, radiation exposure and the need to reside or work in a toxic environment Zeliger, 2016]. SES is also associated with an increased likelihood of having undiagnosed diseases [Bein, et al., 2012; Mielck, et al., 2014; Shaw, et al., 2016].

The detailed OSI lists numerous items that address these points. In the condensed form, all of these are factored into SES as indicated by highest educational level achieved. Educational achievement has been shown to be a valid indicator of SES, with lower SES individuals more likely more likely to lead unhealthy, OS raising life styles and to have undiagnosed diseases [Yin, et al., 2017]. In the condensed OSI form, 5 education levels are identified: some high school, high school graduate, some college, college graduate and graduate degree. These are assigned 5,4,3,2 and 1 OSI point respectively.

SES can also be obtained from annual income information, but asking income information can be considered being nosy and discourage some people from completing the questionnaire. Hence, income is not used in the condensed OSI questionnaire.

\section{G. Residence}

In the $21^{\text {st }}$ century, 90 percent of the world's people, regardless of SES, are exposed to air pollution, as air quality is the same on both sides of the tracks [Combes and Franchineau, 2019; World Health Organization, 2018]. All air pollutants are toxic, raise $\mathrm{OS}$ in a dose response relationship, impact the OSI, and are responsible for the onset of numerous diseases including respiratory diseases, cardiovascular diseases, several cancers and Alzheimer's disease [Combes and Franchineau, 2019; Zhou, et al., 2019; Xia, et al., 2019; Kilian J and Kitazawa M, 2018].

Recently, a method to quantify air pollution impact on disease onset, termed the Air Quality Toxicity Index (AQTI) has been reported [Zeliger, 2020]. This method, based upon the dose response relationship between toxic exposure and OS elevation [Zeliger, 2016 and the numerous references contained therein], enables the classification of air quality for individual locations to be calculated and reported as cumulative annual values.

In America, the United States Environmental Protection Agency (EPA) measures air quality in multiple locations on a daily basis and reports the data on-line daily as the Air Quality Index (AQI) [EPA, 2019]. Worldwide, the World Air Quality Project similarly reports air quality data for hundreds of cities. [World Air Quality Index Project, 2019]. On an annual basis, these indices identify the number of days in which the air quality in a given locale is classified as either good, moderate, unhealthy for sensitive groups, unhealthy, very unhealthy or hazardous. Hazard numbers ranging from $1-6$ have been assigned to the six air quality classifications, as follows [Zeliger, 2020].

\begin{tabular}{lc} 
Air Quality Classification & Hazard Number \\
\hline Good & 1 \\
Moderate & 2 \\
Unhealthy for Sensitive Groups & 3 \\
Unhealthy & 4 \\
Very Unhealthy & 5 \\
Hazardous & 6
\end{tabular}

The air pollution impact on OSI is obtained by multiplying the number of days per year for each of the six EPA classifications at the residence of the responder by the hazard number for that classification and adding the these up to yield the AQTI total as shown in table 3. Also shown in table 3. are the OSI air pollution severities (OSI - AP). These are assigned number values from $0-5$, and entered into the OSI condensed form. It should also be noted that EPA publishes daily and annual air quality data for cities and counties in all U.S. states [EPA, 2019].

\section{H. Chronic Psychological Stress}

Psychological stress, anxiety and depression are associated with most diseases and are more pronounced as illnesses progress [Sahle, et al., 2020]. Psychological stress, anxiety and depression raise OS via the release of hormones that elevate OS. Chronic activation of this stress response system results in disease and triggers numerous health problems [Mayo Clinic, 2019], including:

Anxiety 
Depression

Memory and concentration issues

Digestive problems

Headaches

Heart disease

Sleep problems

Weight gain

Respondents are asked to check if they often feel stressed, anxious or depressed. One point is assigned for each positive response.

The relevance of the OSI to predicting disease onset probability as well as for other applications uses has been previously established [Zeliger, 2017, 2019, 2019a, 2019b, $2019 \mathrm{c}]$. When the OSI condensed form values are used as presented here an excellent correlation between standard form and condensed form OSI values obtains, with condensed form values showing less than a $10 \%$ variation from standard form values.

To sum up, the point values assigned to each of the parameters contained in the OSI condensed questionnaire are as follows:

\begin{tabular}{|c|c|}
\hline Age & 1 for each decade of age starting at age 40 \\
\hline Personal & 0,1 , or 2 , depending upon weight status \\
\hline Diseases & 5 per prevalent disease \\
\hline Medications & $\begin{array}{l}1 \text { per prescribed or over-the-counter } \\
\text { medication regularly taken }\end{array}$ \\
\hline Genetics & 1 per each item checked \\
\hline Education & $\begin{array}{l}5,4,3,2 \text { or } 1 \text {, depending upon highest } \\
\text { education level achieved }\end{array}$ \\
\hline Residence & $\begin{array}{l}0,1,2,3,4 \text {, or } 5 \text {, depending upon air } \\
\text { pollution level at residence }\end{array}$ \\
\hline Stress & $\begin{array}{l}0,1,2 \text { or } 3 \text {, depending upon chronic } \\
\text { psychological status }\end{array}$ \\
\hline
\end{tabular}

The OSI score has been shown to be related to the likelihood of further disease onset as follows [Zeliger, 2017]:

\begin{tabular}{ll} 
OSI Level & Disease Likelihood \\
\hline $0-15$ & Indicative of good health \\
$16-30$ & Disease onset predicted \\
$31-45$ & Disease onset probable \\
46 or higher & Disease imminent
\end{tabular}

Though the OSI can predict the likelihood of disease onset, it cannot predict which disease(s) are likely to strike.

The condensed OSI form has multiple uses, including the following:

- Part of routine medical examinations to alert clinicians to potential illnesses in seemingly healthy people.

- Use as a screening aid in areas with limited medical personnel and facilities.

- Serving to identify lifestyle changes that will lower OS and likelihood of disease onset.

- Taking of public health surveys to identify disease clusters arising from exposures to chronic exposures to toxic chemicals.

- Making community medical need projections including estimating clinical staffing and resource needs.
- $\quad$ Serving as an indicator of the need to have a person fill out the detailed OSI form which can alert clinicians to specific potential problems.

- Researching sequences of disease onset in those with multiple diseases.

- $\quad$ Predicting numbers of people likely to ail with noncommunicative diseases in different geographic areas.

The detailed OSI questionnaire has been modified since it was first introduced. The form currently in use to build a data base has the condensed form questions embedded in it, thus facilitating a comparison of the two forms.

The OSI condensed form does have limitations. It does not probe as yet undiagnosed diseases, conditions and symptoms that are addressed in the detailed form. Also, to date the number of OSI questionnaire responders evaluated is not yet large enough to yield statistically significant data. Work in this area is ongoing, with the understanding that the point values of the condensed form OSI can be adjusted, if necessary, and that additional parameters could be added to the eight reported here, if indicated. Despite the limitations just noted, preliminary results indicate that the condensed OSI form produces values that are 90 percent or more in agreement with that from the detailed OSI form.

\section{CONCLUSIONS}

The premise of this paper is that a questionnaire consisting of eight parameters can be used to determine the OSI and that these are representative of all sources of oxidative stress elevation.

The eight parameters used to calculate the condensed form OSI all raise OS levels in dose response relationships [Zeliger, 2016]. These have all been shown to be additive in predicting disease onset likelihood, demonstrating that multiple causes of the same disease are probable, and that no single cause need be the sole one [Zeliger, 2019].

The condensed form of the OSI is a simplified version of the detailed OSI and that like the detailed OSI, can be used to predict the likelihood of disease onset.

\section{REFERENCES}

Awdeh ZL, Yunis EJ, Auden MJ, Fici C, Pugliese A, Larsen CE, Alper CA. 2006. A genetic explanation for the rising incidence of type 1 diabetes, a polygenic disease. J Autoimmun; 27(3):174-181.

Bein T, Hackner K, Zou T, Schultes S, Bosch T, Schlitt HJ, et al. 2012. Socioeconomic status, severity of disease and level of family members' care in adult surgical intensive care patients: the prospective ECSSTASI study. Intensive Care Med; 38:612-619.

CDC. 2015. Centers for Disease Control and Prevention. Healthy weight. The health effects of overweight and obesity. https://cdc.gov/healthyweight/effects/index.html Accessed January 25,2020 .

CDC. 2018. Centers for Disease Control, National Center for Health Statistics.

https://www.cdc.gov/nchs/hus/contents2018.htm\#Table 021

Cencioni C, Spalotta F, Martelli F, Valente S, Mai A, Zeiher AM, Gaetano C. Oxidative stress and epigenetic regulation in aging and are-related diseases. Int J Mol Sci; 14:17643-17663.

Combes A, Franchineau G. 2019. Fine particle environmental pollution and cardiovascular diseases. Metabolism, 2019. doi: 10.16/j.metabol.2019.07.008 Accessed January 27, 2020.

Dato S, Crocco P, D’Aquila P, de Rango F, Belllizi D, Rose G, Passarino G. 2013. Exploring the role of genetic variability and lifestyle in 
oxidative stress response for healthy aging and longevity, Int $\mathrm{J}$ Mol Sci; 14:16443-16472.

EPA. 2019. Air quality index (AQI) https://airnow.gov/index.cfm?action=aquibasics.aqi Accessed January 2, 2020.

Epel ES, Blackburn EH, Dhabhar FS, Adler NE, Morrow JD, Cawthorn RM. 2014. Accelerated telomere shortening in response to life stress. Proc Nat Acad Sci USA 101(49):17312-17315.

Guillaumet-Adkins A, Yanez Y, Preis MD, Palancia-Ballester C, Sandoval J. 2017.Ox Med Cell Longevity article No. ID9175806. Doi: 10.1155/2017/9175806. Accessed January 23, 2020.

Hou L, Joyce BT, Gao T, Liu L, Zheng Y, Penedo FJ, et al. 2015. Blood telomere length attrition and cancer development in the normative aging study cohort. EBioMedicine 132(6):591-596.

Jiang T, Yu JT, Tian Y, Tan L. 2013. Epidemiology and etiology of Alzheimer's disease. From genetic to non-genetic factors. Curr Alzheimer Res; 9:852-867.

Kelly FJ. 2003. Oxidative stress: Its role in air pollution and adverse health effects. Occup Environ Med 60:612-616.

Kilian J, Kitazawa M. 2018. The emerging risk of exposure to air pollution on cognitive decline and Alzheimer's disease - Evidence from epidemiological and animal studies. Biomed J 41(3):141-162.

Mayo Clinic. 2019. Healthy lifestyle stress management. https://www.mayoclinic.org/heaalthy-lifestyles-management/indepth/stress/art-20046037

Mielck A, Vogelman M, Reiner L. 2014. Health-related quality of life and socioeconomic status: inequalities among adults with a chronic disease. $\quad$ BioMed Central; $12: 58$ http://www.hqlo.com/content/12/1/58. Accessed January 21, 2020.

Riggs DW, Zafar N, Krishnasamy S, Yeager R, Rai, SN, Bhatnagar A, O'Toole TE. 2020. Exposure to airborne fine particulate matter is associated with impaired endothelial function and biomarkers of oxidative stress and inflammation. Environ Res. 2020. doi: 10.1016/j.envres.2019.108890. Accessed December 30, 2019.

Sahle BW, Chen W, Melaku YA, Akombi BJ, Rawal LB, Renzaho AMN 2020. Association of psychosocial factors with risk of chronic diseases: A nationwide longitudinal study. Am J Prev Med; 58(2):e39-e50. Doi: 10.1016/j.amepre.2019.09.007 Accessed January $27,2020$.

Shaw KM, Theis KA, Self-Brown S, Roblin DW, Barker L. 2016. Chronic disease disparities by county economic status and metropolitan classification, behavioral risk factor surveillance system, 2013.
Centers for Disease Control. Preventing chronic disease. https://www.cdc.giv/pcd/issues/2016/16_0088.htm. Accessed January 21, 2020. World Air Quality Index Project. 2019. https://aqicn.com. Accessed January 27, 2020.

Xia SY, Huang DS, Jia H, Zhao Y, Lin N, Mao MQ, et al. 2019. Relationship between atmospheric pollutants and risk of death caused by cardiovascular and respiratory diseases and malignant tumors in Shenyang, China from 2013to 2016: an ecological research. Chin Med J (Engl); 132(19):2269-2277.

Yin H, Wu Q, Cui Y, Hao Y, Liu C, Li Y, et al. 2017. Socioeconomic status and prevalence of chronic non-communicable diseases in Chinese women: a structural equation modelling approach. BMJ Open 2017;7:e014402. doi: 10.1136/bmjopen-2016-014402. Accessed January 22,2020

Zeliger HI. 2016. Predicting disease onset in clinically healthy people. Interdiscip Toxicol; 9(2):39-54.

Zeliger HI. 2017. Oxidative stress index predicts disease onset. J Med Res Prac; 6(3):86-92.

Zeliger HI. 2019. Predicting Alzheimer's disease onset. Eur J Med Health Sci; 1(1). DOI: http:dx.doi.org/10.24018/ejmed.2019.1.1.16 Accessed January 28, 2020.

Zeliger HI. 2019a. Oxidative stress index as a public health survey instrument. Eur J Med Health Sci; 1(2). DOI: http:dx.doi.org/10.24018/ejmed.2019.1.2.28. Accessed January 28, 2020.

Zeliger HI. 2019b. Predicting and reducing adverse drug reactions. Euro J Med Health Sci; 1(4). DOI: http://dx.doi.org/10.24018/ejmed.2019.1.4.91 Accessed January 322, 2020.

Zeliger HI. 2019c. Oxidative stress index: disease onset prediction and prevention. EC Pharm Toxicol 7.9 (2019):1022-1036.

Zeliger HI. 2020. Air quality toxicity index (AQTI): Quantifying air pollution impact on disease onset. Eur J Med Health Sci; 2(1). DOI: http:dx.doi.org/10.24018/ejmed.2020.2.1.143. Accessed January 28, 2020.

Zhou H, Wang T, Zhou F, Liu Y, Zhao W, Wang X, et al. 2019. Ambient air pollution and daily hospital admissions for respiratory disease in children in Guiyang, China. Front Pediatr. 2019. Doi: 10.3389/fped.2019.00400. Accessed January 30, 2020.

Table 1. OSI condensed questionnaire.

Date

No.

1. Age:

2. Personal:

female

male

height weight

Do you work with toxic chemicals or live near a toxic landfill

Yes $\overline{\text { No }}$

3. Diseases:

All diseases and diagnosed conditions you now have.

For cancer, add the stage $(1,2,3$ or 4$)$ if known

1.

4.

7.

2.

Which of these diseases were diagnosed in the last year

4. Medications: Number of medications regularly taken

5. Family history: Number of the above diseases parents had/have

Mother Father

6. Education: Check highest level achieved some high school some college 
graduate school

7. Residence: $\quad$ City State and County where you live.

City __ State___ County

8. Stress: $\quad$ Check any that apply

Do you often feel: Stressed

Anxious

Depressed

Total

Table 2. Detailed OSI questionnaire.

\section{OXIDATIVE STRESS INDEX (OSI) QUESTIONNAIRE}

DATE

NUMBER

GENDER

Female

Male

AGE

EDUCATION Check highest level achieved

some high school some college graduate school

high school graduate college graduate

RESIDENCE City State

County

\section{FAMILY HISTORY}

Put one check for each parent, sibling (sister or brother) or grandparent who has or had each of the diseases or conditions identified.

Parent with neurological disease - Alzheimer's, Parkinson's, Lou Gehrig,

$$
\text { ADHD or Autism }
$$

Parent with diabetes

Parent with heart disease, heart attack or stroke

Parent with asthma or COPD

Parent with allergies - hay fever, animals, food or chemicals

Parent with cancer

Siblings with neurological disease - Alzheimer's, Parkinson's or Lou Gehrig,

$$
\text { ADHD or Autism }
$$

Siblings with diabetes

Siblings with heart disease, heart attack or stroke

Siblings with asthma or COPD

Siblings with allergies - hay fever, animals, food or chemicals

Siblings with cancer

Grandparents with neurological disease - Alzheimer's, Parkinson's or Lou Gehrig, 
ADHD or Autism

Grandparents with diabetes

Grandparents with heart disease, heart attack or stroke

Grandparents with asthma or COPD

Grandparent with allergies - hay fever, animals, food or chemicals

Grandparent with cancer

Total Family History Checks

AGE

Check all age boxes that apply. If, for example, you are 55 years old check the first two boxes. If you're 82, check all 5 of these boxes
40 or older
50 or older
60 or older
70 or older
80 or older

\section{WEIGHT}

Find your healthy weight, on the weight chart (see table 2.2 on page 46). Check all the weight boxes that apply. If, for example you are 40 pounds over your recommended weight, check the first three boxes and add three checks to the total. Find your recommended weight on the weight chart at the end of the OSI checklist.

10 - 20 pounds overweight

21 - 40 pounds overweight

31 - 60 pounds overweight

61 - 80 pounds overweight

81 or more pounds overweight

Total age and weight checks

\section{ILLNESSES and CONDITIONS}

Check all illnesses or conditions that you have been diagnosed with and currently have.

Acne

ADHD (Attention Deficit Hyperactivity Disorder)

AIDS or HIV

Alcohol addiction

Allergic rhinitis (sinus inflammation)

ALS (Lou Gehrig's disease)

Anemia

Anorexia

Anxiety disorder

Arthritis

Asthma

Autism and ASD

Autism or autism spectrum disorder (ASD)

Benign prostate hyperplasia (enlargement - $\mathrm{BPH}$ )

Bipolar disorder

Bronchitis (chronic)

Bulimia

Bulging or herniated disc

Carpal tunnel syndrome 
Cancer - Check all that apply. If stage three, for example, check first three

Cancer - stage 1

Cancer - stage 1 or 2

Cancer - stage 1, 2 or 3

Cancer - stage 1, 2, 3 or 4

Frequent infection

Frequent sinus infections (sinusitis)

Glaucoma

Gout

Gum disease

Heart attack

Heart Disease or heart problems

Hemorrhoids

Hepatitis B (chronic)

High blood pressure (hypertension)

High cholesterol

Herpes

Inflammatory bowl disease

Irritable bowel syndrome (IBS)

Kidney disease

Leukemia

Liver cirrhosis

Liver disease

Lupus

Lyme disease

Macular degeneration

Malaria

Metabolic syndrome (pre-diabetes)

Middle ear infection (frequent)

Migraine headaches

Multiple chemical sensitivity (MCS)

Multiple sclerosis

Obesity

Osteoarthritis

Osteoporosis

Parkinson's disease

Periodontal disease (swollen or bleeding gums)

Post-traumatic stress disorder (PTSD)

Rocky Mountain spotted fever

Psoriasis

Rosacea

Schizophrenia

Sexually transmitted disease (STD)

Shingles

Sjogren's syndrome

Sleep apnea

Stroke

TB (Tuberculosis)

Thyroid disease

Tourette syndrome

Tremors

Ulcers

Varicose veins

West Nile Fever

Yellow fever

Zika

Write in the names of any other illnesses you have that were not listed above and check those. 


\section{Total Disease Checks}

\section{DISEASE START}

How many of the diseases checked above started in

The past 2 years?

The past 5 years?

The past 10 years?

\section{SYMPTOMS}

Check all the symptoms that you currently experience.

Abdominal pain (frequent)

Allergic reactions to chemicals

Allergic reactions to any foods

Allergic reactions to insects

Allergic reactions to medications

Allergic reactions to plants (Hay fever)

Ankle pain

Attention span decline

Anxiety often

Appetite loss

Bleeding gums

Blood in stool

Blood in urine

Blurred or cloudy vision

Bruise easily

Burning when urinating

Butterflies in your stomach often

Change in skin color

Chest pain

Constant chills

Constipation

Cough that is persistent

Coughing or spitting up blood

Decision making difficulties

Decline in learning ability

Decreased eye sight

Decreased sex drive

Diarrhea (frequent)

Difficulty completing familiar tasks

Difficulty concentrating

Difficulty getting warm

Difficulty maintaining balance

Difficulty solving problems

Difficulty swallowing

Difficulty walking

Difficulty concentrating or finding words

Dizziness

Drained of energy

Dreams that are bizarre and recurring

Excessive mucous production

Excessive thirst

Eye discomfort or pain

Eye redness

Fatigue

Feel depressed a lot

Feel less alert or fuzzy headed 
Fever

Food allergies

Foot pain

Foot swelling

Fungal infection such as athlete's food that persists

Frequent anxiety

Frequent depression

Frequent stress

Frequent headaches

Frequent indigestion

Frequent itching

Frequent rashes

Frequent urination

Graying of hair

Hair loss (not due to chemotherapy)

Have itchy scaly skin rashes

Headaches frequently

Hear voices inside you

Hearing loss that comes on suddenly

Heart palpitations (throbbing)

Heartburn

Hip pain

Hoarseness

Increased susceptibility to infections

Indigestion (frequent)

Insomnia

Irregular periods

Itchy hands

Itchy skin other than hands

Jaw pain

Leg swelling

Learning new things more difficult

Light headedness

Long recovery time from infections

Losing track of time

Loss of coordination

Loss of muscle tone

Loss of taste

Lower back pain

Memory loss

Mood swings from very high to very low and vice versa

Mouth sores that don't go away quickly

Muscle aches that last a long time

Muscle cramps

Muscle spasms

Nasal congestion

Nausea

Neck pain

Nervousness

Nightmares regularly

Nose bleeds

Knee pain

Numbness or tingling in hands or feet

Pain in joints

Heart palpitations

Pelvic pain

Perspire (sweat) profusely

Post nasal drip that lingers

Post traumatic stress disorder (PTSD)

Problems finding the words you want 


\section{Rapid hair loss}

Rapid heartbeat

Scaly skin

Seizures

Shortness of breath

Shoulder pain that lingers

Sinus pain

Skin mole growth

Skin rashes

Sleep less than 7 hours per night

Sleep more than 9 hours a night

Slow to heal from cuts, bruises or other injuries

Slurred speech

Smaller field of vision

Sore throat that doesn't heal

Stressed out most or all of the time

Stuffy nose

Swollen eye lids

Tics (involuntary movements)

Tingling in the hands or feet

Tire easily

Tired most of the time

Tooth pain

Tremors

Twitching

Unusual vaginal bleeding or discharge

Urination difficulty

Urination pain

Varicose veins

Vomiting

Wake up more than 3 times per night

Weakness

Weight gain

Weight loss (rapid)

Wheezing

Wrinkling or loss of tone in skin

Yawning frequently

\section{Total Symptoms Checks}

\section{TEST RESULTS}

The following address results obtained from test doctors ordered done as part of annual examinations. Check all that apply to you.

High or low blood sugar

High or low BUN (blood urea nitrogen)

High or low calcium

High or low carbon dioxide (bicarbonate)

High or low chloride

High cholesterol

High or low creatinine

High glucose

High or low potassium

High PSA

High or low sodium

High triglycerides

Low blood oxygen

Low potassium 


\section{Total Test Results Checks}

\section{PRESCRIPTION MEDICINES REGULARLY TAKEN}

Check each of the boxes that apply. If you regularly take five prescription drugs, for example, check all of the first 5 items, so that the total number of items checked equals the total number of prescriptions regularly taken.

\section{1 prescription \\ 2 prescriptions \\ 3 prescriptions \\ 4 prescriptions \\ 5 prescriptions \\ 6 prescriptions \\ 7 prescriptions \\ 8 prescriptions \\ 9 prescriptions}

10 or more prescriptions

Have a heart pacemaker

Total Prescription Medicine Checks

\section{DIET}

Check each item that applies to the foods that are part of your regular eat.

Alcoholic beverages (beer, wine, spirits) more than 1 drink per day

Artificial sweeteners for coffee or tea

Canned or frozen cooked foods regularly eaten (soups, pastas, meats)

Bread and pasta made primarily from white processed flour

Fast food frequently eaten

Fewer than 3 fruits or vegetables a day

Grilled, smoked or blackened meat, chicken or fish

Food high in fat (whole milk, cheeses, foods cooked with butter and animal fat)

Often eat processed foods (bacon, hot dogs, salami, sausages. deli meats

Eat red meat more than 2 times a week

Eat foods high in sugar (sweetened drinks and desserts)

Salty food

\section{Total Diet Checks}

\section{LIFE STYLE}

These items refer to where you live, the type of work you do and chemicals you may be exposed to.

Are a farmer that regularly uses pesticides

Burn wood for heat or for cooking

Constantly use a cell phone

Drink chlorinated water

Drink more than one alcoholic drink per day

Take steroids for muscle enhancement

Exercise less than one half hour a week

Have mold in your home

Have new (less than 6 months old) carpet in your home

Have pets in your home that you are allergic to

Live down wind from a smoking industrial chimney

Live in a city with air quality alerts

Live or work close to a cell tower

Live or work near high voltage electrical transmission lines 
Live near a heavily traveled highway or road

Live near a landfill

Live near industrial storage tanks (chemicals, oil or asphalt)

Live with a smoker

Regularly experience allergic reactions in your home

Regularly experience allergic reactions in your work place

Regularly use room or furniture deodorants

Regularly play contact sports

Tobacco use. Check all of the items that apply. For example, if you smoke one pack of cigarettes a day, check both of the first 2 items. If you smoke two packs a day, check all of the first 4 items.

Smoke 10 cigarettes or less daily (even just one)

Smoke a pack a day (20 cigarettes)

Smoke a pack and a half a day (30 cigarettes)

Smoke two packs a day (40 cigarettes)

Smoke more than two packs a day

Smoke 1-5 cigars a day

Smoke 6 or more cigars a day

Use smokeless tobacco

Work as a toll booth collector

Work in very hot or very cold conditions regularly

Work in an adhesives or coatings manufacturing plant

Work in an agricultural chemical manufacturing plant

Work as an automobile, diesel or aircraft mechanic

Work with chemicals on the job regularly

Work in a dusty environment regularly

Work in a landfill

Work in a hair or nail salon

Work in metal refinery or mill

Work as a miner

Work in a noisy environment

Work in a paint, lacquer, stain or varnish manufacturing plant

Work as a painter

Work as a pilot or flight attendant

Work as a pesticide applicator

Work in a petroleum refinery

Work in a plastics manufacturing plant

Work in a plywood or particle board manufacturing plant

Work in a polluted environment (road paver, toll booth operator, for example)

Work in a water or sewage treatment plant

Work in wood treatment plant

\section{Total Lifestyle Checks}

\section{OSI, Total of All Checks}

Table 3. Annual air quality classification as shown by AQTI.

$\begin{array}{llc}\text { AIR QUALITY CLASSIFICATION } & \text { AQTI } & \text { OSI-AP } \\ \text { Good } & 365-450 & 0 \\ \text { Moderate } & 451-600 & 1 \\ \text { Unhealthy for Sensitive Groups } & 601-750 & 2 \\ \text { Unhealthy } & 751-900 & 3 \\ \text { Very Unhealthy } & 901-1050 & 4 \\ \text { Hazardous } & 1051 \text { or higher } & 5\end{array}$

